\title{
Study of twice-weekly injections of Teriparatide by comparing efficacy with once-weekly injections in osteoporosis patients: the TWICE study
}

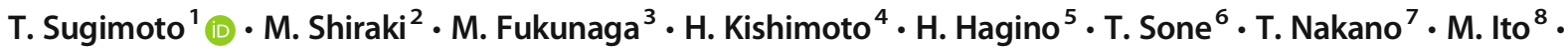 \\ H. Yoshikawa ${ }^{9} \cdot$ T. Minamida $^{10} \cdot$ Y. Tsuruya $^{10} \cdot$ T. Nakamura ${ }^{11}$
}

Received: 15 March 2019 / Accepted: 24 July 2019 /Published online: 8 August 2019

(C) The Author(s) 2019

\begin{abstract}
Summary A 48-week, multicenter, randomized, double-blind, double-dummy, active-controlled, non-inferiority trial (the TWICE study) conducted in Japanese primary osteoporosis patients with a high risk of fractures demonstrated that a 28.2- $\mu \mathrm{g}$ twice-weekly regimen of teriparatide can provide comparable efficacy to a $56.5-\mu \mathrm{g}$ once-weekly regimen of teriparatide, while also improving safety.

Introduction While a 56.5- $\mathrm{gg}$ once-weekly regimen of teriparatide has high efficacy for osteoporosis, treatment continuation rates are low, with one of the major causes being adverse drug reactions such as nausea or vomiting. The TWICE study was therefore conducted to investigate whether a twice-weekly regimen with $28.2-\mu \mathrm{g}$ teriparatide can provide comparable efficacy to the 56.5- $\mu$ g once-weekly regimen while improving safety.

Methods A 48-week, multicenter, randomized, double-blind, double-dummy, active-controlled, non-inferiority trial was conducted in Japan. Patients with primary osteoporosis aged $\geq 65$ years at high risk of fractures $(n=553)$ were randomly allocated to the 28.2- $\mu$ g twice-weekly group $(n=277)$ or the 56.5 - $\mu$ g once-weekly group $(n=276)$. The primary endpoint was the percentage change in lumbar spine (L2-L4) bone mineral density (BMD) at final follow-up.

Results The percentage changes in lumbar spine (L2-L4) BMD at final follow-up in the 28.2- $\mu$ g twice-weekly and 56.5- $\mu \mathrm{g}$ once-weekly groups were $7.3 \%$ and $5.9 \%$, respectively; the difference (95\% confidence interval [CI]) in percentage change was $1.3 \%(0.400-2.283 \%)$. Since the lower limit of the $95 \%$ CI was above the pre-specified non-inferiority margin (-1.6\%), noninferiority of the 28.2- $\mu \mathrm{g}$ twice-weekly group was demonstrated. Adverse drug reactions were significantly less frequent in the $28.2-\mu$ g twice-weekly group $(39.7 \%$ vs $56.2 \%$; $p<0.01)$; the incidence of major adverse drug reactions was lower, and the number of subjects who discontinued due to adverse drug reactions was less in the 28.2- $\mu$ g twice-weekly group.
\end{abstract}

Electronic supplementary material The online version of this article (https://doi.org/10.1007/s00198-019-05111-6) contains supplementary material, which is available to authorized users.

T. Sugimoto

sugimoto@med.shimane-u.ac.jp

1 Internal Medicine 1, Shimane University Faculty of Medicine, 89-1 Ennya-cho, Izumo, Shimane 693-8501, Japan

2 Research Institute and Practice for Involutional Diseases, 1610-1 Misatomeisei, Azumino, Nagano 399-8101, Japan

3 Kawasaki Medical School, 577 Matsushima, Kurashiki, Okayama 701-0192, Japan

4 Nojima Hospital, 2714-1 Sesakimachi, Kurayoshi, Tottori 682-0863, Japan

5 School of Health Science, Tottori University, 86 Nishicho, Yonago, Tottori 683-8503, Japan
6 Department of Nuclear Medicine, Kawasaki Medical School, 577 Matsushima, Kurashiki, Okayama 701-0192, Japan

7 Tamana Central Hospital, 1950 Naka, Tamana, Kumamoto 865-0064, Japan

8 Center for Diversity and Inclusion, Nagasaki University, 1-7-1 Sakamoto, Nagasaki, Nagasaki 852-8520, Japan

9 Department of Orthopaedic Surgery, Graduated School of Medicine, Osaka University, 1-1 Yamadaoka, Suita, Osaka 565-0871, Japan

10 Asahi Kasei Pharma Corporation, 1-1-2 Yurakucho, Chiyoda-ku, Tokyo 100-0006, Japan

11 Toto Sangenjaya Rehabilitation Hospital, 1-24-3 Sangenjaya, Setagaya-ku, Tokyo 154-0024, Japan 
Conclusions A 28.2- $\mu \mathrm{g}$ twice-weekly regimen of teriparatide can provide comparable efficacy to a $56.5-\mu \mathrm{g}$ once-weekly regimen while improving safety.

Clinical trial registration JapicCTI-163477.

Keywords Teriparatide $\cdot$ Osteoporosis $\cdot$ Bone mineral density $\cdot$ Safety $\cdot$ Twice-weekly $\cdot$ Randomized controlled trial

\section{Introduction}

Osteoporosis is a skeletal disease characterized by reduced bone strength that confers an increased susceptibility to fractures [1]. In Japan, the number of patients with osteoporosis has been increasingly yearly as the society ages rapidly, affecting an estimated 12.8 million people at present [2]. In patients with osteoporosis, a minor external force from the loads of daily living or a fall may cause a fracture, which decreases activities of daily living (ADL) and quality of life (QOL) and reportedly leads to decreased life expectancy [3-6].

Treatments for osteoporosis consist of background therapy that includes nutrition and exercise, and aggressive therapy with medication. Teriparatide is a bone-forming stimulating agent and a widely-used treatment option for osteoporosis patients with a high risk of fractures. In Japan, a $20-\mu \mathrm{g}$ teriparatide formulation for daily use and a $56.5-\mu \mathrm{g}$ teriparatide formulation for onceweekly use are available.

A placebo-controlled, 72-week, Teriparatide Once-Weekly Efficacy Research (TOWER) trial conducted in Japanese osteoporosis patients with a high risk of fractures demonstrated that, compared with placebo, a 56.5- $\mu$ g once-weekly regimen showed a powerful fracture-inhibiting effect, providing an $80 \%$ reduction in the relative risk of new vertebral fractures [7]. Moreover, the regimen has also been shown to increase bone mineral density (BMD) substantially [7]. Another clinical study also reported that the $56.5-\mu \mathrm{g}$ once-weekly regimen promotes bone formation without increasing bone resorption, showing a characteristic pattern of bone turnover, and it increases BMD of the radius, which is rich in cortical bone [8]. In addition, a non-clinical study showed that the once-weekly regimen of teriparatide is unlikely to induce histologic changes such as cortical porosity [9].

While the $56.5-\mu \mathrm{g}$ once-weekly regimen of teriparatide has demonstrated high efficacy, it is known to be associated with low rates of treatment continuation [10]. Adverse drug reactions such as nausea or vomiting have been cited as a reason behind the difficulty with treatment continuity. The mechanism by which teriparatide induces nausea or vomiting has not yet been fully elucidated, due to the existence of complex mechanisms in both central and local effects [11], and no uniform method of managing nausea and vomiting has been established. We decided to address this issue by reducing the incidence of such adverse drug reactions in an attempt to improve treatment continuity. In a previous dose-finding study, once-weekly regimens using
$28.2 \mu \mathrm{g}$ and $14.1 \mu \mathrm{g}$ were associated with incidences of adverse drug reactions that were comparable but lower than seen with the $56.5-\mu \mathrm{g}$ once-weekly regimen of teriparatide [12]. In terms of efficacy, however, the $28.2-\mu \mathrm{g}$ once-weekly regimen and the $14.1-\mu \mathrm{g}$ once-weekly regimen were inferior to the $56.5-\mu \mathrm{g}$ once-weekly regimen [12]. Meanwhile, a non-clinical study showed that, regardless of the dose, efficacies are practically the same as long as a comparable weekly exposure to teriparatide is achieved [13]. We hypothesized that a $28.2-\mu \mathrm{g}$ twice-weekly regimen of teriparatide can still achieve comparable efficacy provided by the $56.5-\mu \mathrm{g}$ once-weekly regimen and reduce the incidence of adverse drug reactions. To confirm the justification for a $28.2-\mu \mathrm{g}$ twice-weekly regimen, a clinical pharmacological study of 6-week treatment was conducted in healthy Japanese postmenopausal women. The results showed that changes in bone turnover markers were comparable between the $28.2-\mu \mathrm{g}$ twiceweekly regimen and the $56.5-\mu \mathrm{g}$ once-weekly regimen, and the $28.2-\mu \mathrm{g}$ twice-weekly regimen was superior to the $56.5-\mu \mathrm{g}$ onceweekly regimen in terms of safety [14]. Nevertheless, the efficacy and safety of the dosage and administration have yet to be confirmed in patients with osteoporosis.

We thus conducted this study of twice-weekly injections of teriparatide by comparing efficacy with once-weekly injections in patients with osteoporosis (the TWICE study) to verify whether the $28.2-\mu \mathrm{g}$ twice-weekly regimen of teriparatide can provide comparable efficacy to the $56.5-\mu$ g once-weekly regimen of teriparatide, while also improving safety, in Japanese primary osteoporosis patients at high risk of fractures.

\section{Patients and methods}

\section{Study design}

This was a 48-week, multicenter, randomized, double-blind, double-dummy, active-controlled, non-inferiority trial conducted in Japan (JapicCTI-163477). The subjects were randomly allocated to a group receiving teriparatide $28.2 \mu \mathrm{g}$ twice weekly and placebo once weekly $(28.2-\mu \mathrm{g}$ twiceweekly group) or a group receiving teriparatide $56.5 \mu \mathrm{g}$ once weekly and placebo twice weekly $(56.5-\mu \mathrm{g}$ once-weekly group) in a 1:1 ratio by dynamic allocation based on the minimization method using sex, age, and young adult mean (\%) of 
BMD of the lumbar spine at enrollment as randomization factors. The 28.2- $\mu \mathrm{g}$ twice-weekly regimen of teriparatide and the twice-weekly regimen of placebo were selfadministered with an autoinjector by the subjects, in principle, every 3 or 4 days (with 2 or 3 days between injection days). The $56.5-\mu \mathrm{g}$ once-weekly regimen of teriparatide and the once-weekly regimen of placebo were administered by physicians. The subjects also received concomitant treatment with daily oral calcium $610 \mathrm{mg}$, vitamin $\mathrm{D}_{3} 400 \mathrm{IU}$, and magnesium $30 \mathrm{mg}$ (Shin Calcichew $\mathrm{D}_{3}$, Takeda Consumer Healthcare Company Limited, Osaka, Japan). This study was conducted in accordance with the ethical principles of the Declaration of Helsinki and Good Clinical Practice (GCP). Moreover, institutional review board approval was in place before the study at each study site.

\section{Subjects}

The eligibility criteria for study subjects were as follows: patients aged 65 years or older, able to walk independently, diagnosis of primary osteoporosis based on the diagnostic criteria for primary osteoporosis (FY2012 revised version) [15], at least one but no more than five prevalent fractures between the fourth thoracic vertebra (Th4) and the fourth lumbar vertebra (L4), mean BMD of the second through fourth lumbar vertebrae (L2-L4) of less than $80 \%$ of the young adult mean at the time of enrollment, and the ability to self-administer injections. Patients excluded were those with a diagnosis of secondary osteoporosis, with a non-osteoporotic disease that causes decreased bone mass, with any X-ray findings that affect the assessment of lumbar BMD by dual energy X-ray absorptiometry (DXA), with a serum calcium level $\geq 11.0 \mathrm{mg} / \mathrm{dl}$, with a malignant bone tumor or a metastatic bone tumor, with previous radiation therapy affecting the bone or otherwise at high risk of developing osteosarcoma, or with a serum alkaline phosphatase (ALP) level more than double the standard level. In addition, patients who were otherwise judged by the investigator as unsuitable for participation in the clinical study, or who had received treatment with teriparatide or an anti-receptor activator of nuclear factor kappa B ligand (RANKL) antibody in the past, bisphosphonate within 52 weeks before treatment commencement, or any other osteoporosis drug within 8 weeks before treatment commencement were also excluded. Recruitment was conducted from January to July 2017.

\section{Efficacy endpoints}

The primary endpoint selected was the percentage change in lumbar spine (L2-L4) BMD at the final time point. The secondary endpoints were the percentage changes in total hip BMD and femoral neck BMD, incidence of fractures, and the time profiles of bone turnover markers (bone formation and bone resorption markers).

\section{Efficacy measures}

BMDs of the lumbar spine and femur were measured by DXA at screening, baseline, and weeks 24 and 48. DXA was performed with a Discovery, Explorer, Horizon (Hologic, Marlborough, MA), Lunar DPX, Lunar iDXA, or Lunar Prodigy (GE Healthcare, Chicago, IL) device, which was calibrated before each test for precision control with a lumbar spine phantom attached to the device. For external quality control (QC), specialists checked QC sheets from all study sites every month and performed maintenance as needed. The lumbar and femoral BMD measurements were analyzed centrally by a BMD analysis laboratory, and whether a datum was to be included or excluded or warranted re-analysis was evaluated centrally in accordance with the criteria established in advance by a Data Review Committee for BMD assessment.

To assess morphological vertebral fractures, $\mathrm{X}$-ray images of the thoracic/lumbar vertebrae were taken at screening, baseline, and weeks 24 and 48. To avoid inter-assessor variability, a Data Review Committee for fracture assessment conducted central assessments of the vertebrae from Th4 through L4 for fractures by a semi-quantitative method (SQ) that involved vertebral measurement $[16,17]$. The presence or absence of vertebral fractures was determined by comparing X-ray images of thoracic/lumbar vertebrae taken at baseline and after treatment, based on the percentage reduction in vertebral height determined by vertebral measurements. A vertebral fracture was defined as a change from baseline after treatment in grade of a vertebral body combined with a $20 \%$ or greater reduction in height at the posterior edge, center, or anterior edge of the vertebral body. A vertebral body that was normal at baseline but became deformed after treatment was considered a new vertebral fracture, and a vertebral body that was deformed at baseline and became further deformed after treatment was considered a worsened vertebral fracture.

Non-vertebral fractures were confirmed by the investigator when appropriate. A fragility fracture was defined as a fracture that the investigator judged to be not induced by a large external force. Major non-vertebral fragility fractures due to osteoporosis were defined as fragility fractures: hip fracture, rib fracture, pelvic fracture, proximal humerus fracture, distal radius fracture, or lower leg fracture.

To measure bone turnover markers, samples were collected at baseline and before the investigational product was administered at weeks 4, 12, 24, and 48. Samples were stored either in a refrigerator or a freezer, depending on the type of marker to be measured, before measurements were performed collectively by a validated laboratory (LSI Medience Corporation, Tokyo, Japan). Serum osteocalcin (OC) was measured by 
fluoroimmunoassay (Tosoh Corporation, Tokyo, Japan); serum type I procollagen-N-propeptide (P1NP) was measured by electrochemiluminescence immunoassay (ECLIA) (Roche Diagnostics K.K., Tokyo, Japan); urinary type I collagen cross-linked N-telopeptide (NTX) was measured by enzyme immunoassay (Alere Medical Co., Ltd., Tokyo, Japan); and serum type I collagen cross-linked C-telopeptide (CTX) was measured by ECLIA (Roche Diagnostics K.K.).

\section{Adverse events}

Safety was assessed collectively on the basis of all adverse events, including those that were serious and those leading to study discontinuation. The subjects underwent medical examinations and regular blood tests, blood biochemistry tests, urinalyses, and vital sign measurements. Vital signs were measured before and $10 \mathrm{~min}$ and approximately $1 \mathrm{~h}$ after investigational product administration at weeks $0,4,12,24$, and 48 .

The investigators reported adverse events, which were coded to the preferred terms in the Medical Dictionary for Regulatory Activities (MedDRA, version 21.0).

\section{Sample size}

The 56.5- $\mu$ g once-weekly regimen of teriparatide prescribed for the comparator group has been confirmed to be highly effective in inhibiting fractures, relative to placebo, in the phase 3 doubleblind, parallel group TOWER trial of 72-week treatment [7]. In the TOWER trial, the regimen provided a high-level fractureinhibiting effect sustained throughout the treatment period and increased BMD over time [7]. Furthermore, the percentage change in lumbar spine (L2-L4) BMD at the final time point up to week 48 in the TOWER trial was $5.3 \%$ in subjects who received the $56.5-\mu \mathrm{g}$ once-weekly regimen and $0.5 \%$ in those who received placebo, showing a difference in percentage change (95\% confidence interval [CI]) of 4.8\% (3.9-5.8\%) between the $56.5-\mu \mathrm{g}$ once-weekly regimen and placebo [7]. With that, a non-inferiority margin of $2.4-1.6 \%$, equivalent to $1 / 2$ to $1 /$ 3 of the percentage difference of $4.8 \%$ was considered justified for this study.

Assuming a difference of $0 \%$ in the point estimate of percentage change in lumbar spine (L2-L4) BMD between the $28.2-\mu \mathrm{g}$ twice-weekly group and the $56.5-\mu \mathrm{g}$ once-weekly group, a standard deviation of $5.0 \%$ in each group, a conservative non-inferiority margin of $1.6 \%$, a detection power of $90 \%$, and a significance level of one-sided $2.5 \%$, the sample size required to establish non-inferiority was 207 subjects per group. Furthermore, based on the sizes of the lumbar spine (L2-L4) BMD analysis set at baseline and the final time point in the TOWER trial, the percentage of dropouts and exclusions from bone assessment was estimated to be $14 \%$. With that, a sample size of 500 subjects overall, 250 per group, was selected to initiate treatment.

\section{Statistical analysis}

Efficacy was analyzed using the full analysis set (FAS). The FAS included all subjects who received the investigational product, except those who had any GCP deviation, those confirmed to have no osteoporosis, and those for whom no posttreatment efficacy data were available.

The primary analysis verified the non-inferiority of the $28.2-\mu \mathrm{g}$ twice-weekly group to the $56.5-\mu \mathrm{g}$ once-weekly group. This was demonstrated by the fact that the lower bound of a two-sided $95 \%$ confidence interval for the difference in the percentage change in lumbar spine (L2-L4) BMD at the final time point was greater than $-1.6 \%$ (non-inferiority margin).

BMDs and bone turnover markers were analyzed, with percentage changes from baseline summarized over time. Fractures were analyzed with incidences of fractures tabulated.

The safety analysis set included all subjects who received the investigational product. Incidences of each adverse event were summarized. Statistical analyses were performed using SAS version 9.4 (SAS Institute, Cary, NC, USA). Percentage changes from baseline in BMD (at weeks 24 and 48 and the final time point) were compared by Student's $t$ test, and percentage changes from baseline in bone turnover markers (at weeks $4,12,24$, and 48) were compared by the Wilcoxon rank sum test. Incidences of adverse events were compared by Fisher's exact test and the odds ratios of adverse events (the $28.2-\mu \mathrm{g}$ twice-weekly group vs. the 56.5- $\mu$ g once-weekly group) were calculated. All statistical tests were performed with a significance level of 0.05 .

Clinically significant abnormalities in vital signs were defined as " $\leq 90 \mathrm{mmHg}$ and decrease $\geq 20 \mathrm{mmHg}$ from baseline" for systolic blood pressure, " $\leq 50 \mathrm{mmHg}$ and decrease $\geq 15 \mathrm{mmHg}$ from baseline" for diastolic blood pressure, and " $\geq 120 \mathrm{bpm}$ and increase $\geq 15 \mathrm{bpm}$ from baseline" for pulse rate [18].

\section{Results}

\section{Subjects}

Of the 859 subjects at 92 sites throughout Japan who gave informed consent, 553 patients with primary osteoporosis aged 65 years or older with a high risk of fractures were randomly allocated: 277 to the $28.2-\mu$ g twice-weekly group and 276 to the $56.5-\mu \mathrm{g}$ once-weekly group. All 553 subjects received treatment with the investigational product, with $242(87.4 \%)$ in the $28.2-\mu \mathrm{g}$ twice-weekly group and $235(85.1 \%)$ in the $56.5-\mu \mathrm{g}$ once-weekly group completing treatment. Thirty-five subjects $(12.6 \%)$ in the $28.2-\mu$ g twice-weekly group and $41(14.9 \%)$ in the $56.5-\mu \mathrm{g}$ once-weekly group withdrew from the study, with 
fewer withdrawals in the 28.2- $\mu \mathrm{g}$ twice-weekly group than in the 56.5- $\mu$ g once-weekly group (Fig. 1).

Two subjects in the 28.2- $\mu \mathrm{g}$ twice-weekly group were excluded from the FAS because one had no efficacy data and the other took unallocated study drugs. Baseline subject characteristics differed little between the two groups (Table 1).

\section{Bone mineral density}

The percentage change in lumbar spine (L2-L4) BMD at the final time point was $7.3 \%$ in the $28.2-\mu \mathrm{g}$ twice-weekly group and $5.9 \%$ in the $56.5-\mu \mathrm{g}$ once-weekly group, showing a difference $(95 \% \mathrm{CI})$ in percentage change of $1.3 \%(0.400$ $2.283 \%$ ) between the two groups. Given that the lower limit of the $95 \%$ CI was above the non-inferiority margin $(-1.6 \%)$ specified in advance, non-inferiority of the $28.2-\mu \mathrm{g}$ twiceweekly group to the $56.5-\mu \mathrm{g}$ once-weekly group was demonstrated (Fig. 2a).

Moreover, the percentage change from baseline in lumbar spine (L2-L4) BMD was 5.0\% in the 28.2- $\mu$ g twiceweekly group and $3.8 \%$ in the $56.5-\mu$ g once-weekly group after 24 weeks, and $7.5 \%$ and $6.0 \%$, respectively, after 48 weeks, showing a significantly greater change $(p<0.01)$ in the $28.2-\mu$ g twice-weekly group than in the
56.5- $\mu$ g once-weekly group at each evaluation time point (Fig. 2a, Sup. Fig. 1a, Sup. Table 1).

The percentage change in total hip BMD was $1.3 \%$ in the $28.2-\mu \mathrm{g}$ twice-weekly group and $1.0 \%$ in the $56.5-\mu \mathrm{g}$ onceweekly group after 24 weeks; $1.8 \%$ and $1.3 \%$, respectively, after 48 weeks; and $1.8 \%$ and $1.3 \%$, respectively, at the final time point (Fig. 2b, Sup. Fig. 1b, Sup. Table 1). The percentage change in femoral neck BMD was $1.6 \%$ in the $28.2-\mu \mathrm{g}$ twice-weekly group and $1.0 \%$ in the $56.5-\mu$ g once-weekly group after 24 weeks; $2.1 \%$ and $1.4 \%$, respectively, after 48 weeks; and $2.0 \%$ and $1.4 \%$, respectively, at the final time point (Fig. 2c, Sup. Fig. 1c, Sup. Table 1). The percentage changes in total hip BMD and femoral neck BMD were great$\mathrm{er}$ in the $28.2-\mu \mathrm{g}$ twice-weekly group than in the $56.5-\mu \mathrm{g}$ once-weekly group at each evaluation time point, though the differences were not significant (Fig. 2b, c).

\section{Fractures}

The cumulative number of subjects with new vertebral fractures after 48 weeks was three in the $28.2-\mu \mathrm{g}$ twice-weekly group and four in the 56.5- $\mu$ g once-weekly group. The incidence of new vertebral fractures during each interval between evaluations was $1.2 \%$ (3/259 subjects) in the $28.2-\mu \mathrm{g}$ twice-weekly group and $1.2 \%(3 / 247)$ in the $56.5-\mu$ g once-weekly group in the first

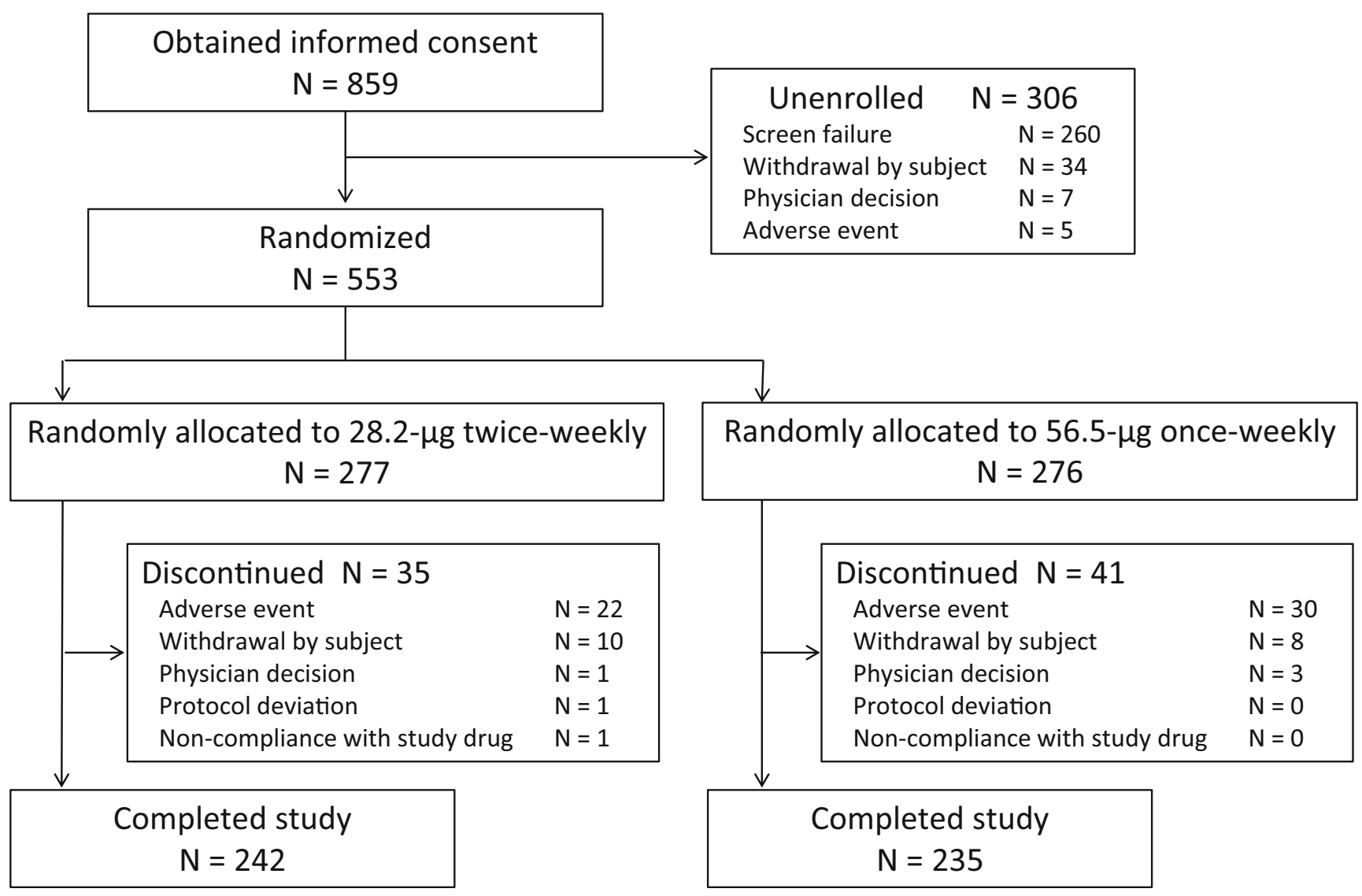

Fig. 1 Patient disposition 
Table 1 Subjects' baseline characteristics

\begin{tabular}{lll}
\hline Variable & $28.2-\mu$ g twice-weekly $(N=275)$ & $56.5-\mu$ g once-weekly $(N=276)$ \\
\hline Age (y) & $74.1 \pm 5.9$ & $74.5 \pm 6.0$ \\
Sex (female), $n(\%)$ & $252(91.6)$ & $251(90.9)$ \\
Time since menopause (y) & $24.1 \pm 7.0(N=252)$ & $25.0 \pm 7.4(N=251)$ \\
Height (cm) & $151.12 \pm 6.64$ & $150.78 \pm 6.42$ \\
Weight $(\mathrm{kg})$ & $50.18 \pm 7.72$ & $51.23 \pm 7.51$ \\
Body mass index $\left(\mathrm{kg} / \mathrm{m}^{2}\right)$ & $21.97 \pm 3.07$ & $22.54 \pm 3.08$ \\
Prevalent vertebral fractures, $n(\%)$ & & \\
0 & $48(17.5)$ & $40(14.5)$ \\
1 & $131(47.6)$ & $144(52.2)$ \\
2-3 & $80(29.1)$ & $76(27.5)$ \\
$4-5$ & $14(5.1)$ & $9(3.3)$ \\
No bone assessment & $2(0.7)$ & $7(2.5)$ \\
Lumbar spine BMD T-score & $-2.9 \pm 0.7(N=267)$ & $-2.9 \pm 0.7(N=263)$ \\
Total hip BMD T-score & $-2.3 \pm 0.9(N=272)$ & $-2.2 \pm 0.8(N=271)$ \\
Femoral neck BMD T-score & $-3.1 \pm 0.9(N=272)$ & $-2.9 \pm 0.8(N=271)$ \\
25-OH vitamin D $(\mathrm{ng} / \mathrm{mL})$ & $25.58 \pm 6.62$ & $26.90 \pm 7.15$ \\
Serum osteocalcin $(\mathrm{ng} / \mathrm{mL})$ & $19.67 \pm 9.99(N=268)$ & $19.48 \pm 9.39(N=267)$ \\
Serum P1NP $(\mu \mathrm{g} / \mathrm{L})$ & $52.93 \pm 27.24(N=268)$ & $51.13 \pm 24.27(N=267)$ \\
u-NTX (nmol BCE/mmol Cr) & $53.37 \pm 31.86(N=268)$ & $51.02 \pm 25.60(N=267)$ \\
s-CTX (ng/mL) & $0.367 \pm 0.192(N=268)$ & $0.366 \pm 0.173(N=267)$ \\
\hline
\end{tabular}

Data are expressed as means $\pm \mathrm{SD}$ or numbers of subjects (\%)

$B C E$ bone collagen equivalents, $C r$ creatinine
24 weeks, and $0.0 \%(0 / 244)$ and $0.4 \%(1 / 235)$, respectively, in the next 24 weeks up to week 48 . No subjects in the $28.2-\mu \mathrm{g}$ twice-weekly group had fractures after week 24 .

The cumulative number of subjects with worsened vertebral fractures after 48 weeks was 1 in both groups.

The cumulative number of subjects with major nonvertebral fragility fractures due to osteoporosis after 48 weeks was 8 in the $28.2-\mu \mathrm{g}$ twice-weekly group and 6 in the $56.5-\mu \mathrm{g}$ once-weekly group.

\section{Bone turnover markers}

Results for bone turnover markers, including serum OC, serum P1NP, urinary NTX, and serum CTX, are shown in Fig. 3. The mean percentage change in serum OC levels in both groups increased after treatment and peaked after 4 weeks (Fig. 3a). Thereafter, the level in both groups decreased gradually but remained at levels above baseline up to week 48 . The mean percentage change in serum P1NP levels in both groups increased after treatment and peaked after 4 weeks (Fig. 3b). Thereafter, the level in both groups decreased but remained at levels above baseline up to week 48. The mean percentage change in urinary NTX levels in both groups fluctuated at levels below baseline after treatment and recovered to approximately the baseline level after 48 weeks (Fig. 3c). The mean percentage change in serum CTX levels fluctuated at levels below baseline after treatment and up to week 48 (Fig. 3d). As shown, while the levels of bone formation markers increased after treatment, those of bone resorption markers fluctuated at levels below baseline after treatment. With respect to concentration-time profiles of bone formation markers and bone resorption markers, small differences were noted between the two groups, indicating that the 28.2- $\mu$ g twiceweekly regimen promotes bone formation without increasing bone resorption, just as the $56.5-\mu \mathrm{g}$ once-weekly regimen.

\section{Adverse events and tolerability}

Small differences in incidences of adverse events were noted between the two groups (Table 2). The incidence of adverse drug reactions was significantly lower $(p<0.01)$ in the 28.2- $\mu \mathrm{g}$ twice-weekly group than in the 56.5- $\mu$ g onceweekly group. Adverse drug reactions occurring at an incidence of $5 \%$ or above, in descending order, were as follows: nausea (56 subjects, $20.2 \%$ ), malaise (26 subjects, $9.4 \%$ ), vomiting (25 subjects, $9.0 \%$ ), headache (16 subjects, $5.8 \%)$, and injection site hemorrhage (14 subjects, $5.1 \%$ ) in the $28.2-\mu \mathrm{g}$ twice-weekly group; and nausea (88 subjects, $31.9 \%$ ), vomiting (36 subjects, $13.0 \%$ ), malaise (33 subjects, $12.0 \%$ ), headache (29 subjects, $10.5 \%$ ), and pyrexia (18 subjects, $6.5 \%$ ) in the $56.5-\mu$ g once-weekly group. Nausea, headache, and pyrexia occurred at a 
(a)

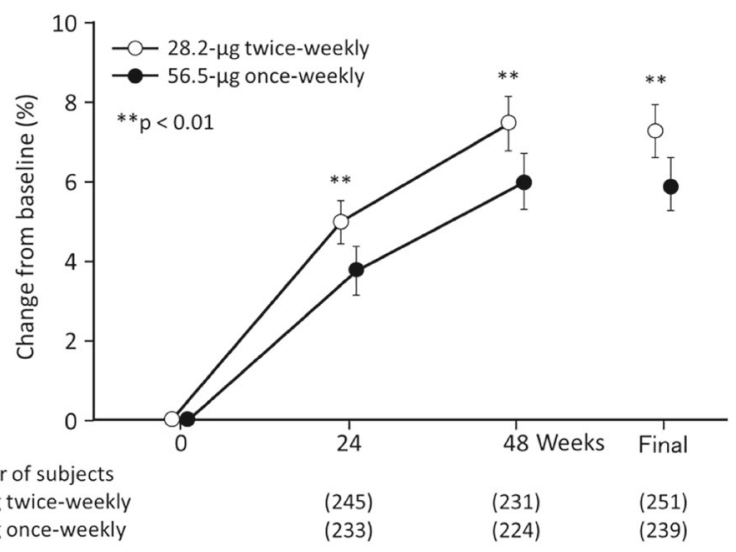

(c)

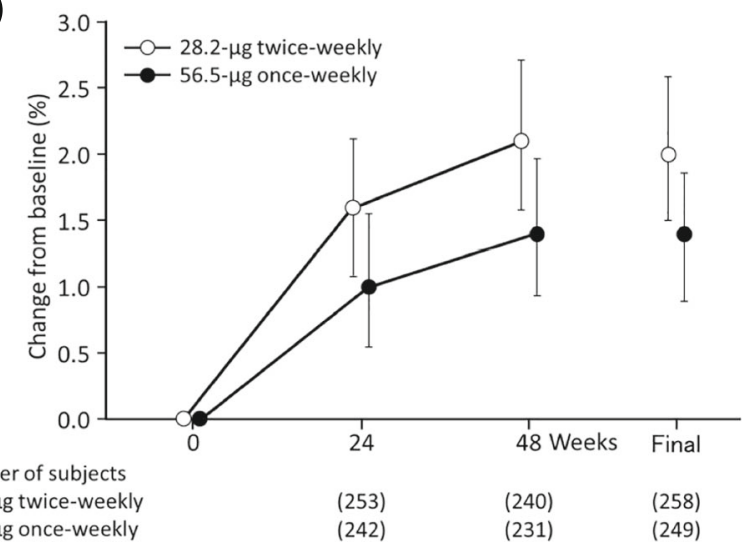

Fig. 2 Mean percentage change from baseline in BMD a lumbar spine (L2-L4), b total hip, and $\mathbf{c}$ femoral neck. Bars indicate 95\% confidence intervals. A comparison was performed using Student's $t$ test. Asterisk (*)

significantly lower incidence in the $28.2-\mu \mathrm{g}$ twice-weekly group than in the $56.5-\mu \mathrm{g}$ once-weekly group.

One subject died (respiratory acidosis) during the study; the subject was assigned to the $56.5-\mu \mathrm{g}$ once-weekly group. The cause of death was deemed to be cervical spinal cord injury and respiratory muscle paralysis as a result of an accidental fall down stairs; the investigator ruled out any causal relationship to the investigational product.

Small differences in incidences of serious adverse events were noted between the two groups. Serious adverse drug reactions occurred in 1 subject $(0.4 \%)$ in the $28.2-\mu \mathrm{g}$ twiceweekly group and in 4 subjects $(1.4 \%)$ in the $56.5-\mu$ g onceweekly group. The events were constipation in 1 subject in the 28.2- $\mu \mathrm{g}$ twice-weekly group and dehydration, headache, sinus node dysfunction, interstitial lung disease, choking sensation, and wrist fracture in 1 subject each in the $56.5-\mu \mathrm{g}$ onceweekly group. Incidences of serious adverse drug reactions differed little between the two groups, and times of onset showed no specific trends.

Incidences of adverse events leading to discontinuation of the study (excluding serious adverse events) tended to be lower in the $28.2-\mu \mathrm{g}$ twice-weekly group. Adverse drug reactions (b)

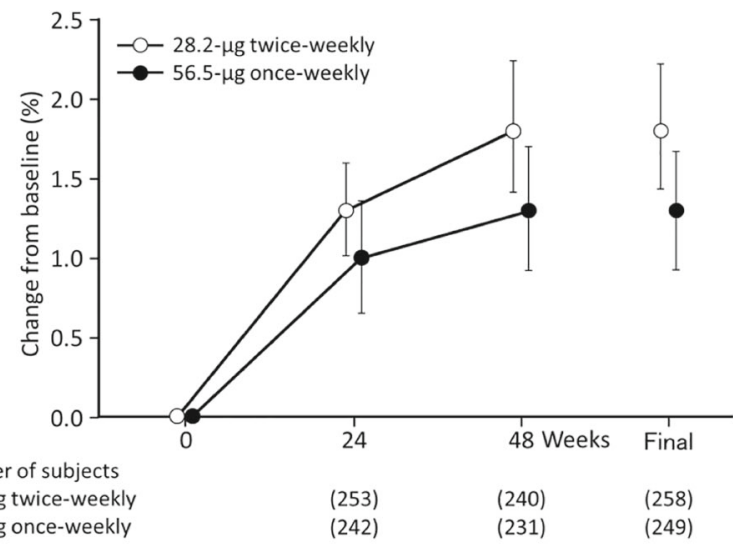

indicates $p<0.05$ versus 56.5 - $\mu \mathrm{g}$ once-weekly; Two asterisks (**) indicate $p<0.01$ versus $56.5-\mu \mathrm{g}$ once-weekly. Open circles, 28.2- $\mu \mathrm{g}$ twiceweekly; closed circles, 56.5 - $\mu$ g once-weekly

leading to discontinuation of the study occurred in 11 subjects $(4.0 \%)$ in the $28.2-\mu \mathrm{g}$ twice-weekly group and in 24 subjects $(8.7 \%)$ in the $56.5-\mu \mathrm{g}$ once-weekly group; the incidence tended to be lower in the 28.2- $\mu$ g twice-weekly group. Adverse events leading to discontinuation of the study that occurred in 3 or more subjects were nausea (6 subjects, $2.2 \%$ ) and headache (3 subjects, $1.1 \%$ ) in the $28.2-\mu$ g twiceweekly group and nausea (12 subjects, $4.3 \%)$, vomiting (5 subjects, $1.8 \%$ ), pyrexia (4 subjects, $1.4 \%$ ), headache ( 3 subjects, $1.1 \%$ ), and feeling abnormal (3 subjects, $1.1 \%$ ) in the 56.5- $\mu$ g once-weekly group, showing that nausea, vomiting, and pyrexia occurred at a lower incidence in the $28.2-\mu \mathrm{g}$ twice-weekly group than in the 56.5- $\mu$ g once-weekly group.

\section{Vital signs}

It should be noted that teriparatide formulations are known to decrease blood pressure when administered and may lead to a transient decrease in blood pressure or loss of consciousness, among other events [19-21]. In the present study, the number of subjects who experienced clinically significant low systolic blood pressure and diastolic blood pressure in the 28.2- $\mu \mathrm{g}$ 
(a)

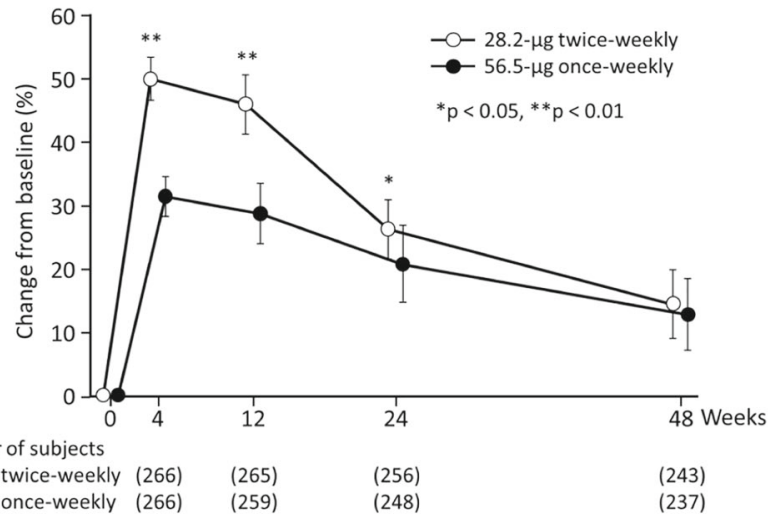

(c)

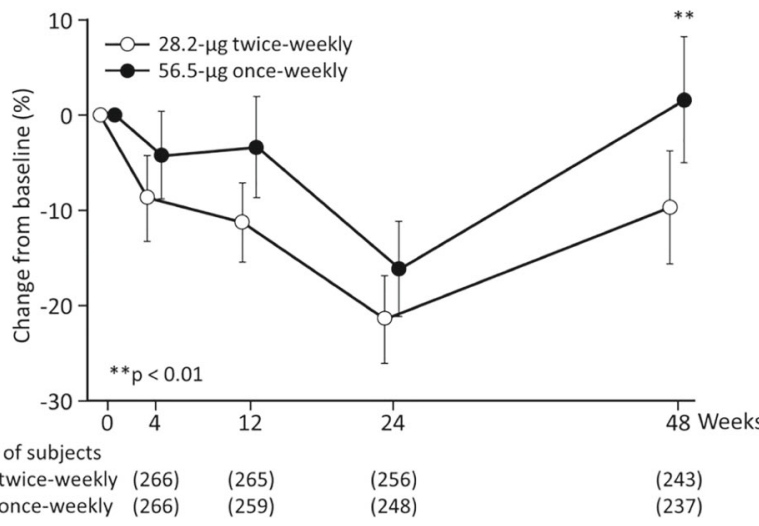

Fig. 3 Mean percentage change from baseline in bone turnover markers a serum-OC, $\mathbf{b}$ serum-P1NP, $\mathbf{c}$ urine-NTX, and $\mathbf{d}$ serum-CTX Bars indicate $95 \%$ confidence intervals. A comparison was performed using the

twice-weekly group was lower than or equivalent to that in the $56.5-\mu$ g once-weekly group (Table 3 ). Zero or one subject experienced a clinically significant high pulse rate in each group at each time point (Table 3). Box plots of changes from pre-dose in systolic blood pressure, diastolic blood pressure, and pulse rate are shown in Supplementary Fig. 2.

An adverse event associated with shock, decreased blood pressure, or loss of consciousness occurred in 3 subjects $(1.1 \%)$ in the $28.2-\mu$ g twice-weekly group and in 9 subjects $(3.3 \%)$ in the $56.5-\mu$ g once-weekly group, showing a lower incidence in the 28.2- $\mu$ g twice-weekly group. In both groups, the most common such event was decreased blood pressure, occurring in 2 subjects $(0.7 \%)$ in the $28.2-\mu$ g twice-weekly group and in 7 subjects $(2.5 \%)$ in the $56.5-\mu$ g once-weekly group, showing a lower incidence in the 28.2- $\mu \mathrm{g}$ twiceweekly group. No adverse events associated with shock occurred in either group (data not shown).

\section{Discussion}

The present study was conducted to determine whether comparable efficacy to that provided by the $56.5-\mu \mathrm{g}$ teriparatide onceweekly regimen and improved safety can be achieved with half
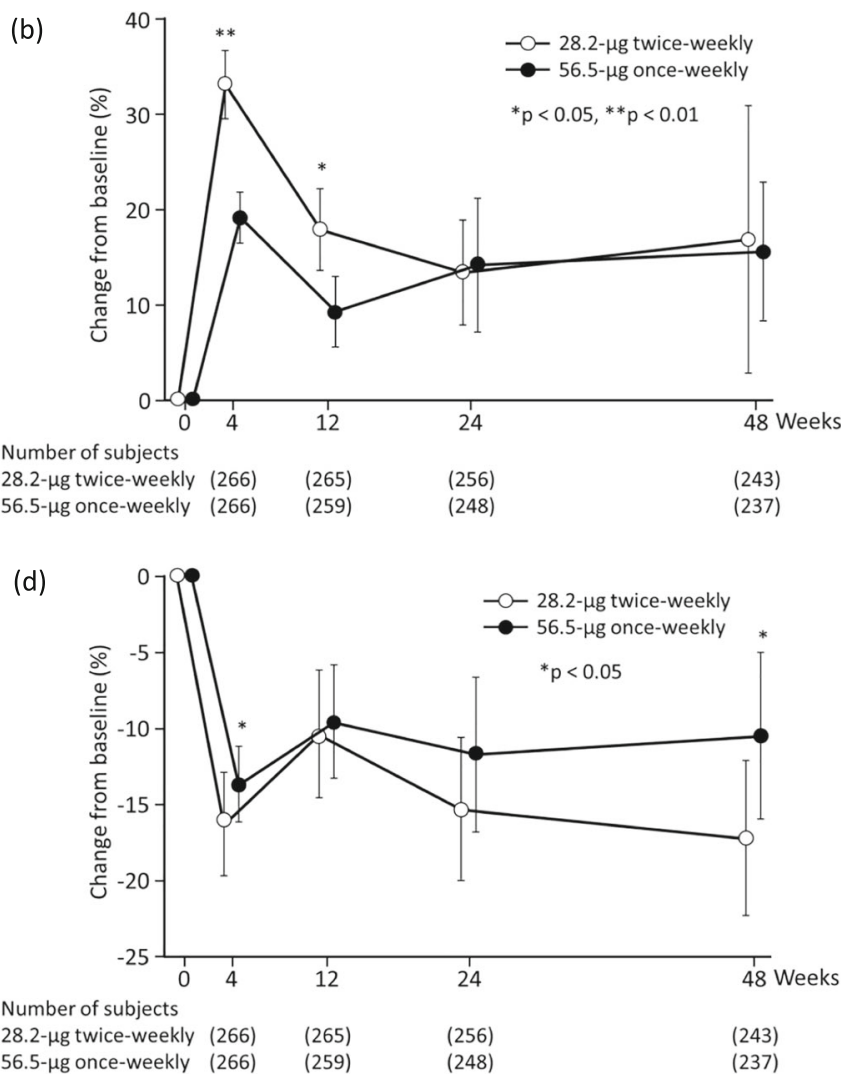

Wilcoxon rank-sum test. Asterisk indicates $(*) p<0.05$ versus $56.5-\mu \mathrm{g}$ once-weekly; Two asterisks (**) $p<0.01$ versus $56.5-\mu$ g once-weekly. Open circles, 28.2- $\mu$ g twice-weekly; closed circles, $56.5-\mu \mathrm{g}$ once-weekly

of the dose (28.2- $\mu$ g teriparatide) given twice weekly. In fact, the data from the present study demonstrated the non-inferiority of the 28.2- $\mu$ g twice-weekly regimen to the $56.5-\mu$ g once-weekly regimen with respect to percentage change in lumbar spine (L2L4) BMD. Furthermore, concentration-time profiles of bone turnover markers showed that the 28.2- $\mu \mathrm{g}$ twice-weekly regimen promotes bone formation without increasing bone resorption, just as the 56.5- $\mathrm{gg}$ once-weekly regimen. A non-clinical study showed that teriparatide administered at low frequencies only increased bone formation, rather than both bone formation and resorption, thereby showing a potential for avoiding cortical porosity $[9,22]$. A 104-week clinical study of the 56.5- $\mu$ g onceweekly regimen confirmed a significant increase in BMD at the radial distal 1/10 site and an increasing trend in BMD at the distal $1 / 3$ and $1 / 6$ sites, which are rich in cortical bone, which suggested that the 56.5- $\mu$ g once-weekly regimen may have achieved the increase in radial BMD by avoiding cortical porosity [8]. Thus, the 28.2- $\mu \mathrm{g}$ twice-weekly regimen is expected to increase BMD substantially while maintaining these characteristics without cortical porosity of the 56.5 - $\mu$ g once-weekly regimen.

In the present study, the percentage change from baseline in lumbar spine (L2-L4) BMD was significantly higher in the $28.2-\mu$ g twice-weekly group than in the $56.5-\mu$ g once-weekly group at all evaluation time points, including weeks 24 and 48 
Table 2 Incidence of adverse events

\begin{tabular}{|c|c|c|c|c|}
\hline Variable & $28.2-\mu$ g twice-weekly $(N=277)$ & $56.5-\mu$ g once-weekly $(N=276)$ & $P$ value & Odds ratio $(95 \% \mathrm{CI})$ \\
\hline Any AE & $245(88.4)$ & $246(89.1)$ & 0.8929 & $0.93(0.55,1.58)$ \\
\hline Any adverse drug reactions & $110(39.7)$ & $155(56.2)$ & 0.0001 & $0.51(0.37,0.72)$ \\
\hline Nausea & $56(20.2)$ & $88(31.9)$ & 0.0019 & $0.54(0.37,0.80)$ \\
\hline Vomiting & $25(9.0)$ & $36(13.0)$ & 0.1377 & $0.66(0.39,1.13)$ \\
\hline Malaise & $26(9.4)$ & $33(12.0)$ & 0.3386 & $0.76(0.44,1.31)$ \\
\hline Headache & $16(5.8)$ & $29(10.5)$ & 0.0443 & $0.52(0.28,0.98)$ \\
\hline Pyrexia & $3(1.1)$ & $18(6.5)$ & 0.0007 & $0.16(0.05,0.54)$ \\
\hline Injection site hemorrhage & $14(5.1)$ & $11(4.0)$ & 0.6831 & $1.28(0.57,2.88)$ \\
\hline Death & $0(0.0)$ & $1(0.4)$ & 0.4991 & - \\
\hline Any SAE & $20(7.2)$ & $24(8.7)$ & 0.5343 & $0.82(0.44,1.52)$ \\
\hline $\begin{array}{l}\text { AE leading to discontinuation } \\
\text { of the study (excluding SAE) }\end{array}$ & $16(5.8)$ & $27(9.8)$ & 0.0829 & $0.57(0.30,1.07)$ \\
\hline
\end{tabular}

Data are expressed as numbers of subjects (\%). Adverse drug reactions occurring at an incidence of $5 \%$ or above are listed

$A E$ adverse event, $S A E$ serious adverse event

and the final time point. The percentage change in the level of bone formation markers was significantly higher in the $28.2-\mu \mathrm{g}$ twice-weekly group than in the 56.5- $\mu$ g onceweekly group, with respect to serum OC, at weeks 4,12 , and 24, and with respect to serum P1NP, at weeks 4 and 12 . These data suggest that the 28.2- $\mu$ g twice-weekly regimen might be superior for promoting bone formation starting from the early phase of treatment and may provide a higher rate of BMD increase than what the 56.5- $\mu \mathrm{g}$ once-weekly regimen can offer. Nevertheless, the mechanism remains unknown and should be further investigated.

While the 56.5- $\mu$ g once-weekly regimen has demonstrated high efficacy, it is known to be associated with low rates of treatment continuation [10]. Adverse drug reactions such as nausea or vomiting have been cited as one of the major reasons behind the difficulty with treatment continuation. In the present study, the incidence of adverse drug reactions was significantly lower in the $28.2-\mu \mathrm{g}$ twice-weekly group than in the $56.5-\mu \mathrm{g}$ once-weekly group, and the incidence of major adverse drug reactions (nausea, vomiting, malaise, headache, and pyrexia) was lower in the 28.2- $\mu$ g twice-weekly group than in the $56.5-\mu \mathrm{g}$ once-weekly group. Furthermore, the incidence of adverse drug reactions leading to discontinuation of the study also tended to be lower in the 28.2- $\mu$ g twice-weekly group. These data thus indicate that halving the dose alleviates adverse drug reactions such as nausea or vomiting and contributes to

Table 3 Incidence of clinically significant abnormalities in vital signs

\begin{tabular}{|c|c|c|c|c|c|}
\hline & & $N$ & $\begin{array}{l}\text { Systolic blood pressure } \\
\leq 90 \mathrm{mmHg} \text { and decrease } \\
\geq 20 \mathrm{mmHg} \text { from baseline }\end{array}$ & $\begin{array}{l}\text { Diastolic blood pressure } \\
\leq 50 \mathrm{mmHg} \text { and decrease } \\
\geq 15 \mathrm{mmHg} \text { from baseline }\end{array}$ & $\begin{array}{l}\text { Pulse rate } \geq 120 \\
\mathrm{bpm} \text { and increase } \geq 15 \mathrm{bpm} \\
\text { from baseline }\end{array}$ \\
\hline \multirow[t]{2}{*}{$0 \mathrm{w}$} & 28.2- $\mu$ g twice-weekly & 276 & $2(0.7)$ & $4(1.4)$ & $1(0.4)$ \\
\hline & 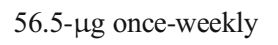 & 275 & $6(2.2)$ & $4(1.5)$ & $0(0.0)$ \\
\hline \multirow[t]{2}{*}{$4 \mathrm{w}$} & $28.2-\mu$ g twice-weekly & 266 & $0(0.0)$ & $7(2.6)$ & $0(0.0)$ \\
\hline & 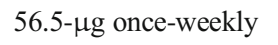 & 266 & $4(1.5)$ & $8(3.0)$ & $0(0.0)$ \\
\hline \multirow[t]{2}{*}{$12 \mathrm{w}$} & $28.2-\mu \mathrm{g}$ twice-weekly & 263 & $2(0.8)$ & $4(1.5)$ & $1(0.4)$ \\
\hline & 56.5- $\mu$ g once-weekly & 253 & $3(1.2)$ & $4(1.6)$ & $0(0.0)$ \\
\hline \multirow[t]{2}{*}{$24 \mathrm{w}$} & 28.2- $\mu$ g twice-weekly & 251 & $1(0.4)$ & $3(1.2)$ & $1(0.4)$ \\
\hline & 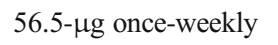 & 244 & $3(1.2)$ & $9(3.7)$ & $0(0.0)$ \\
\hline \multirow[t]{2}{*}{$48 \mathrm{w}$} & $28.2-\mu \mathrm{g}$ twice-weekly & 241 & $3(1.2)$ & $3(1.2)$ & $0(0.0)$ \\
\hline & 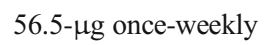 & 233 & $1(0.4)$ & $9(3.9)$ & $1(0.4)$ \\
\hline
\end{tabular}

Data are expressed as numbers of subjects $(\%)$ with clinically significant abnormalities in vital signs. Vital signs were measured before and 10 min and approximately $1 \mathrm{~h}$ after investigational product administration at weeks $0,4,12,24$, and 48 . A clinically significant abnormality in systolic blood pressure is defined as " $\leq 90 \mathrm{mmHg}$ and decrease $\geq 20 \mathrm{mmHg}$ from baseline". A clinically significant abnormality in diastolic blood pressure is defined as " $\leq 50 \mathrm{mmHg}$ and decrease $\geq 15 \mathrm{mmHg}$ from baseline". A clinically significant abnormality in pulse rate is defined as " $\geq 120 \mathrm{bpm}$ and increase $\geq 15 \mathrm{bpm}$ from baseline" 
improving treatment continuation. In a study of the $56.5-\mu \mathrm{g}$ once-weekly regimen, the lumbar spine (L2-L4) BMD increased over time to week 104 [8]. There is also a report that $83 \%$ of vertebral fractures (Freedman's method) can be explained by a change in lumbar spine BMD [23]. In addition, the 56.5- $\mu \mathrm{g}$ once-weekly regimen is known to be associated with a relatively high rate of treatment discontinuation due to adverse drug reactions starting from the early phase of treatment $[8,10]$. Thus, the improved safety and treatment continuation with the $28.2-\mu \mathrm{g}$ twice-weekly regimen raises the likelihood that patients will continue treatment with teriparatide for the maximum treatment duration, suggesting that patients will be able to benefit from greater therapeutic efficacy.

The $56.5-\mu \mathrm{g}$ once-weekly regimen requires patients to make outpatient visits. The $28.2-\mu \mathrm{g}$ twice-weekly regimen, on the other hand, has been developed to allow self-administration. It will reduce the burden of making outpatient visits on patients and their family members and has the potential of becoming a new therapeutic option for patients who have difficulty making the once-weekly outpatient visits to receive the 56.5- $\mu$ g once-weekly regimen. Another existing teriparatide formulation that permits self-administration is used for the $20-\mu \mathrm{g}$ daily regimen of teriparatide. The selfadministered formulation for the $28.2-\mu \mathrm{g}$ twice-weekly regimen involves less frequent injections, uses a disposable autoinjector that requires no replacement needles, and is thus expected to simplify the self-administration and to improve safety and hygiene.

The limitations of this study include the fact that the study conditions did not allow confirmation of fractures, because the primary endpoint was the percentage change from baseline in lumbar spine BMD, and that the study period and sample size were limited. Nevertheless, as described above, it has been reported that $83 \%$ of vertebral fractures can be explained by a change in lumbar spine BMD [23]. In addition, the incidence of new vertebral fractures in this study was lower than that up to week 48 in the placebo group of the TOWER trial, which differed little from this study in terms of baseline subject characteristics [7], indicating that new vertebral fractures were inhibited in both groups of the present study.

\section{Conclusion}

The 28.2- $\mu$ g twice-weekly regimen of teriparatide provided comparable efficacy to the 56.5- $\mu$ g once-weekly regimen of teriparatide. Halving the dose also reduced incidences of events such as nausea or vomiting to levels below those seen with the 56.5- $\mu$ g once-weekly regimen, confirming improvements in safety and treatment continuation.

The 28.2- $\mu \mathrm{g}$ twice-weekly regimen has been developed as a formulation that reduces patient burden by allowing selfadministration and reducing the frequency of outpatient visits compared with the 56.5- $\mu$ g once-weekly regimen. Moreover, compared with another existing teriparatide formulation that permits self-administration, the new self-administered formulation involves less frequent injections and is even easier to selfadminister.

The results of the present study suggest that the $28.2-\mu \mathrm{g}$ twice-weekly regimen is a new treatment option that improves osteoporosis treatment continuation and outcomes.

Acknowledgments The authors would like to thank the investigators and clinical sites in Japan that participated in this study.

Funding The study was sponsored and funded by the Asahi Kasei Pharma Corporation, Tokyo, Japan. The sponsor had responsibility for quality control. The corresponding author had full access to all of the data in the study and had responsibility for the decision to submit for publication.

The study was jointly designed by the authors and the sponsor, Asahi Kasei Pharma Corporation. The authors discussed the interpretation of the data and the conclusions of the manuscript with the sponsor. Data analyses for publication were the responsibilities of the sponsor. ToS has received research grants from Astellas Pharma, Eisai, Daiichi-Sankyo, Chugai Pharmaceutical, and Eli Lilly Japan, as well as consulting and/ or lecture fees from Asahi Kasei Pharma and Daiichi-Sankyo. MS has received consulting fees from Asahi Kasei Pharma, Chugai Pharmaceutical and Teijin Pharma. HK has received consulting fees from Asahi Kasei Pharma, Chugai Pharmaceutical, Daiichi-Sankyo and EA Pharma. HH has received consulting fees for this study from Asahi Kasei Pharma and lecture fees or grants outside the submitted work from Asahi Kasei Pharma, Astellas Pharma, Chugai Pharmaceutical, Eisai, Eli Lilly Japan, Mitsubishi Tanabe Pharma, Mochida Pharmaceutical, Ono Pharmaceutical, Pfizer, Taisho Toyama Pharmaceutical, Takeda Pharmaceutical, Teijin Pharma and Daiichi-Sankyo. TeS has received research grants from Asahi Kasei Pharma, Astellas Pharma, Kissei Pharmaceutical, Pfizer, Taisho Toyama Pharmaceutical and Takeda Pharmaceutical. TeN has received consulting fees from Asahi Kasei Pharma. MI has received research grants from Asahi Kasei Pharma, Astellas Pharma, Chugai Pharmaceutical and Daiichi-Sankyo. TM is an employee of Asahi Kasei Pharma. YT is an employee of Asahi Kasei Pharma. ToN has received consulting fees from Asahi Kasei Pharma and Chugai Pharmaceutical, and speaker fees from Daiichi-Sankyo and Taisho Toyama Pharmaceutical.

\section{Compliance with ethical standards}

\section{Conflicts of interest None.}

Ethical approval All procedures performed in studies involving human participants were in accordance with the ethical standards of the institutional and/or national research committee and with the 1964 Helsinki declaration and its later amendments or comparable ethical standards.

Informed consent Informed consent was obtained from all individual participants included in the study.

Open Access This article is distributed under the terms of the Creative Commons Attribution-NonCommercial 4.0 International License (http:// creativecommons.org/licenses/by-nc/4.0/), which permits any noncommercial use, distribution, and reproduction in any medium, provided you give appropriate credit to the original author(s) and the source, provide a link to the Creative Commons license, and indicate if changes were made. 


\section{References}

1. NIH Consensus Development Panel on Osteoporosis prevention, diagnosis, and therapy (2001) Osteoporosis prevention, diagnosis, and therapy. JAMA 285:785-795

2. Orimo H, Nakamura T, Hosoi T, Iki M, Uenishi K, Endo N, Ohta H, Shiraki M, Sugimoto T, Suzuki T, Soen S, Nishizawa Y, Hagino H, Fukunaga M, Fujiwara S (2012) Japanese 2011 guidelines for prevention and treatment of osteoporosis-executive summary. Arch Osteoporos 7:3-20

3. Bliuc D, Nguyen ND, Milch VE, Nguyen TV, Eisman JA, Center JR (2009) Mortality risk associated with low-trauma osteoporotic fracture and subsequent fracture in men and women. JAMA 301: 513-521

4. Johnell O, Kanis JA, Odén A, Sernbo I, Redlund-Johnell I, Petterson C, De Laet C, Jönsson B (2004) Mortality after osteoporotic fractures. Osteoporos Int 15:38-42

5. Hasserius R, Karlsson MK, Nilsson BE, Redlund-Johnell I, Johnell O (2003) Prevalent vertebral deformities predict increased mortality and increased fracture rate in both men and women: a 10-year populationbased study of 598 individuals from the Swedish cohort in the European vertebral osteoporosis study. Osteoporos Int 14:61-68

6. Chrischilles EA, Butler CD, Davis CS, Wallace RB (1991) A model of lifetime osteoporosis impact. Arch Intern Med 151:2026-2032

7. Nakamura $T$, Sugimoto $T$, Nakano $T$, Kishimoto $H$, Ito $M$, Fukunaga M, Hagino H, Sone T, Yoshikawa H, Nishizawa Y, Fujita T, Shiraki M (2012) Randomized teriparatide [human parathyroid hormone (PTH) 1-34] once-weekly efficacy research (TOWER) trial for examining the reduction in new vertebral fractures in subjects with primary osteoporosis and high fracture risk. J Clin Endocrinol Metab 97:3097-3106

8. Sugimoto T, Shiraki M, Fukunaga M, Hagino H, Sone T, Nakano T, Kishimoto H, Ito M, Yoshikawa H, Kishida M, Irie C, Nakamura T (2017) 24-month open-label teriparatide once-weekly efficacy research trial examining bone mineral density in subjects with primary osteoporosis and high fracture risk. Adv Ther 34:1727-1740

9. Yamane H, Takakura A, Shimadzu Y, Kodama T, Lee JW, Isogai Y, Ishizuya T, Takao-Kawabata R, Iimura T (2017) Acute development of cortical porosity and endosteal naïve bone formation from the daily but not weekly short-term administration of PTH in rabbit. PLoS One 12:e0175329

10. Usui T, Funagoshi M, Seto K, Ide K, Tanaka S, Kawakami K (2018) Persistence of and switches from teriparatide treatment among women and men with osteoporosis in the real world: a claims database analysis. Arch Osteoporos 13:54

11. Shingaki T, Katayama Y, Nakaoka T, Takashima T, Onoe K, Okauchi T, Hayashinaka E, Wada Y, Cui Y, Watanabe Y (2016) Exploration of antiemetics for osteoporosis therapy-induced nausea and vomiting using PET molecular imaging analysis to gastrointestinal pharmacokinetics. Pharm Res 33:1235-1248
12. Fujita $\mathrm{T}$, Inoue $\mathrm{T}$, Morii $\mathrm{H}$, Morita R, Norimatsu $\mathrm{H}$, Orimo $\mathrm{H}$, Takahashi HE, Yamamoto K, Fukunaga M (1999) Effect of an intermittent weekly dose of human parathyroid hormone (1-34) on osteoporosis: a randomized double-masked prospective study using three dose levels. Osteoporos Int 9:296-306

13. Takakura A, Lee JW, Hirano K, Isogai Y, Ishizuya T, TakaoKawabata R, Iimura T (2017) Administration frequency as well as dosage of PTH are associated with development of cortical porosity in ovariectomized rats. Bone Res 5:17002

14. Kumagai Y, Ose A, Tanaka K, Sugimoto T (2019) Safety profiles, pharmacokinetics, and changes in bone turnover markers after twice-weekly subcutaneous administration of teriparatide in healthy Japanese postmenopausal women: a single-blind randomized study. Clin Pharmacol Drug Dev. https://doi.org/10.1002/cpdd.687

15. Soen S, Fukunaga M, Sugimoto T et al (2013) Diagnostic criteria for primary osteoporosis: year 2012 revision. J Bone Miner Metab 31:247-257

16. Genant HK, Wu CY, van Kuijk CM, Nevitt MC (1993) Vertebral fracture assessment using a semiquantitative technique. J Bone Miner Res 8:1137-1148

17. Hayashi Y, Kushida K, Kitazawa A, Tanizawa T, Hotokebuchi T, Hagino H, Murai H, Taneichi H (1998) Measurement of vertebral body dimensions of the thoracic and lumbar spines of 242 healthy women. J Bone Miner Metab 16:27-33

18. Klepper MJ, Cobert B (2010) Drug safety data: how to analyze, summarize and interpret to determine risk. Jones \& Bartlett Learning, Burlington, pp 143-152

19. Mok LL, Nickols GA, Thompson JC, Cooper CW (1989) Parathyroid hormone as a smooth muscle relaxant. Endocr Rev 10:420-436

20. Enishi $\mathrm{T}$, Uemura $\mathrm{H}$, Katoh $\mathrm{S}$, Inatsugi $\mathrm{M}$, Minato $\mathrm{S}$, Inatsugi $\mathrm{K}$, Inatsugi M, Sato N, Siryo K (2015) Transient severe hypotension with once-weekly subcutaneous injection of teriparatide in osteoporotic patient: a case report and insight for the drug interaction between hypotensive agents and teriparatide. J Med Investig 62:93-96

21. World Health Organization (2018) Teriparatide: shock and loss of consciousness. WHO Drug Information 32:18

22. Yamamoto T, Hasegawa T, Sasaki M, Hongo H, Tsuboi K, Shimizu T, Ota M, Haraguchi M, Takahata M, Oda K, Luiz de Freitas PH, Takakura A, Takao-Kawabata R, Isogai Y, Amizuka N (2016) Frequency of teriparatide administration affects the histological pattern of bone formation in young adult male mice. Endocrinology 157:2604-2620

23. Tanaka S, Kuroda T, Sugimoto T, Nakamura T, Shiraki S (2014) Changes in bone mineral density, bone turnover markers, and vertebral fracture risk reduction with once weekly teriparatide. Curr Med Res Opin 30:931-936

Publisher's note Springer Nature remains neutral with regard to jurisdictional claims in published maps and institutional affiliations. 